\title{
Electron-Beam Fabrication of Nanostructures in Glasses
}

\author{
Nan Jiang
}

Department of Physics, Arizona State University, Tempe, Arizona 98287-1504

Transparency is one of the most prominent properties of glasses (except for metallic glasses). Technologically, the benefits of glass transparency range from simple windows for buildings to glass fibers for ultra-long-distance communications. Oxide glasses typically have a broad transmission window in the visible, while the region of transparency is shifted into the mid-infrared for chalcogenide and into the ultraviolet for fluoride glasses. Due to the rapid development of optical communications and requirement for solar energy, micro/nanopatterning and lithography in transparent materials becomes one of the most deserving research topics for introducing new functionalities of glass in electronic applications and nanostructures. Structural and chemical modification is a fascinating phenomenon of high-energy electrons and interactions in transparent materials. The interaction is confined to within such a small volume. Therefore, focused electron beam can efficiently and precisely deposit energy into a nanometer-sized volume and thus induce localized structural and chemical changes in the glasses.

Glasses are thermodynamically metastable phases. Usually, they have lower density than the corresponding crystals [1], and thus the atoms in the glasses do not pack as densely as in the corresponding crystalline forms. Although this is not necessary to result in the susceptibility of glasses to electron beam, one may expect that the displacement energies for atoms in glasses may be relatively low. In addition, it is quite flexible to add various species into glass matrix to form desired composition. Therefore, the glass is also a good candidate for the fabrication of nanostructure.

Figure 1a shows annular dark-field (ADF) images of the rings formed in $\mathrm{GeO}-\mathrm{SiO}_{2}$ by a STEM probe as a function of exposure time [2]. The diameter of the ring depends on the exposure time, but not on specimen thickness, as shown in Fig. 1b. However, the size of the ring does not increase further if the exposure time exceeds 10 seconds. Then the width of the wall shows little change with the increase of exposure time, remaining at about $3.0 \sim 4.0 \mathrm{~nm}$. The relative height (ADF intensity) of the rings also does not change with the exposure time. Sub-nanometer resolution electron energy loss spectroscopy (EELS) indicates that the composition is $\mathrm{SiO}_{2}$-like inside the ring, with more Ge concentration in the rings, compared to the original glass. In the same specimen, two remarkably uniform nanowires can be created by scanning a STEM probe along a line, as shown in Fig. 1c [3]. The width of these nanowires is about $4 \mathrm{~nm}$, and they are separated by about $20 \mathrm{~nm}$, which is about 100 times bigger than that of $0.2-0.3 \mathrm{~nm}$ electron probe diameter. It is noticed that the $20 \mathrm{~nm}$ spacing is much larger than the probe broadening or sample drift. Spatially resolved EELS analysis reveals that the band gaps and the intensities of the band gap states on the wires are different from those of original glass, and from the region between two nanowires as well.

Precipitation of nanocrystals in the glass can also be controlled by high energy electron beam. As shown in Fig.2, the precipitation of $\mathrm{Ga}_{2} \mathrm{O}_{3}$ nanoparticles is induced by electron irradiation, without thermal treatment [4]. From a microstructural point of view, the diversification of the gallium silicate glass by fast electron irradiation is the same as that occurs in thermal treatments. The $\mathrm{Ga}_{2} \mathrm{O}_{3}$ precipitated by fast electron irradiation results from ionization and field-enhanced migration. The electric field created by electron irradiation greatly enhances the mobility of Ga ions within the illuminated area, and this result in phase separation into Si rich and poor regions. 


\section{References}

[1] e.g., the density of quartz is about $2.62 \mathrm{~g} / \mathrm{cm}^{3}$ while it is about $2.20 \mathrm{~g} / \mathrm{cm}^{3}$ for fused silica.

[2] N. Jiang, et al., Appl. Phys. Lett. 83, 551 (2003).

[3] N. Jiang, et al., Appl. Phys. Lett. 80, 2005 (2002).

[4] N. Jiang, et al., Appl. Phys. Lett. 90, 161909 (2007).

[5] This work is supported by NSF DMR0603993. The use of facilities within the center for solid state science at ASU is also acknowledged.
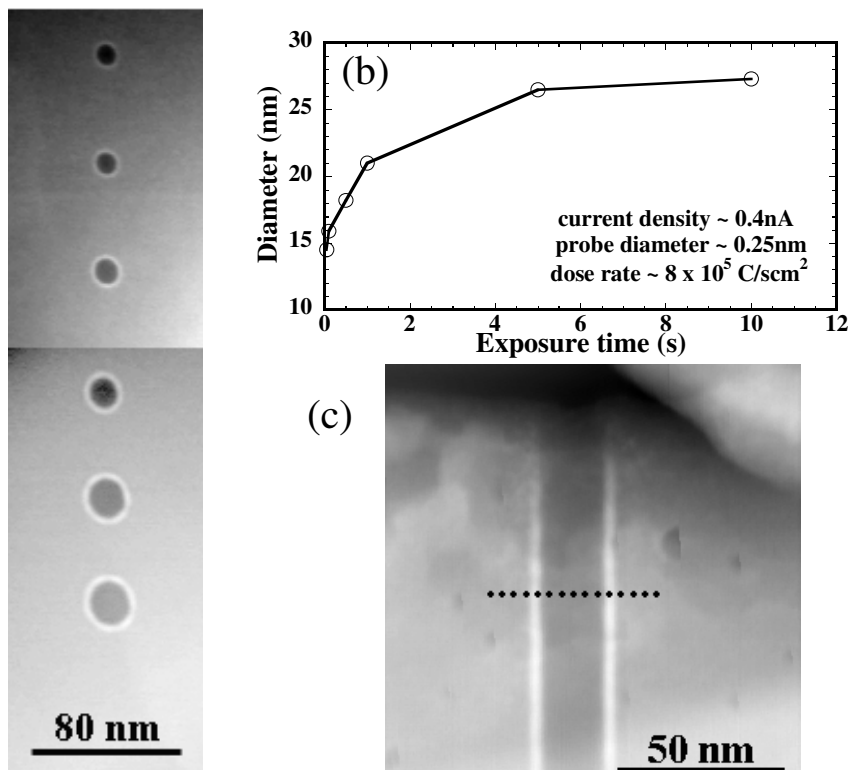

(a)

(c)

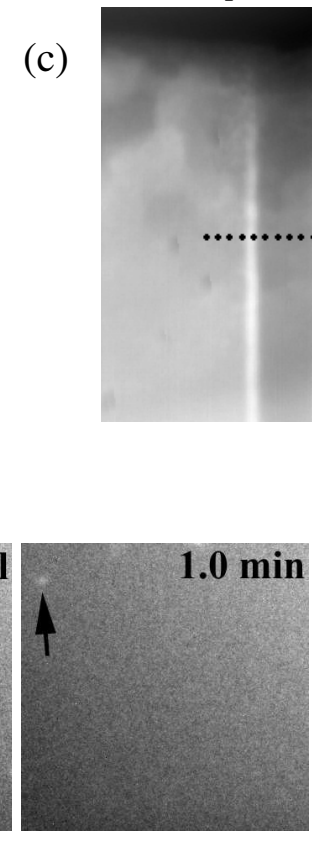

$50 \mathrm{~nm}$
Figure1 (a) Nanorings created by STEM probe in the $10 \mathrm{GeO}_{2}-90 \mathrm{SiO}_{2}$ glass. (b) The dependence of diameters of nanorings on exposure time. (c) Nanowires created by scanning a STEM probe across the specimen of $10 \mathrm{GeO}_{2}-90 \mathrm{SiO}_{2}$ glass.
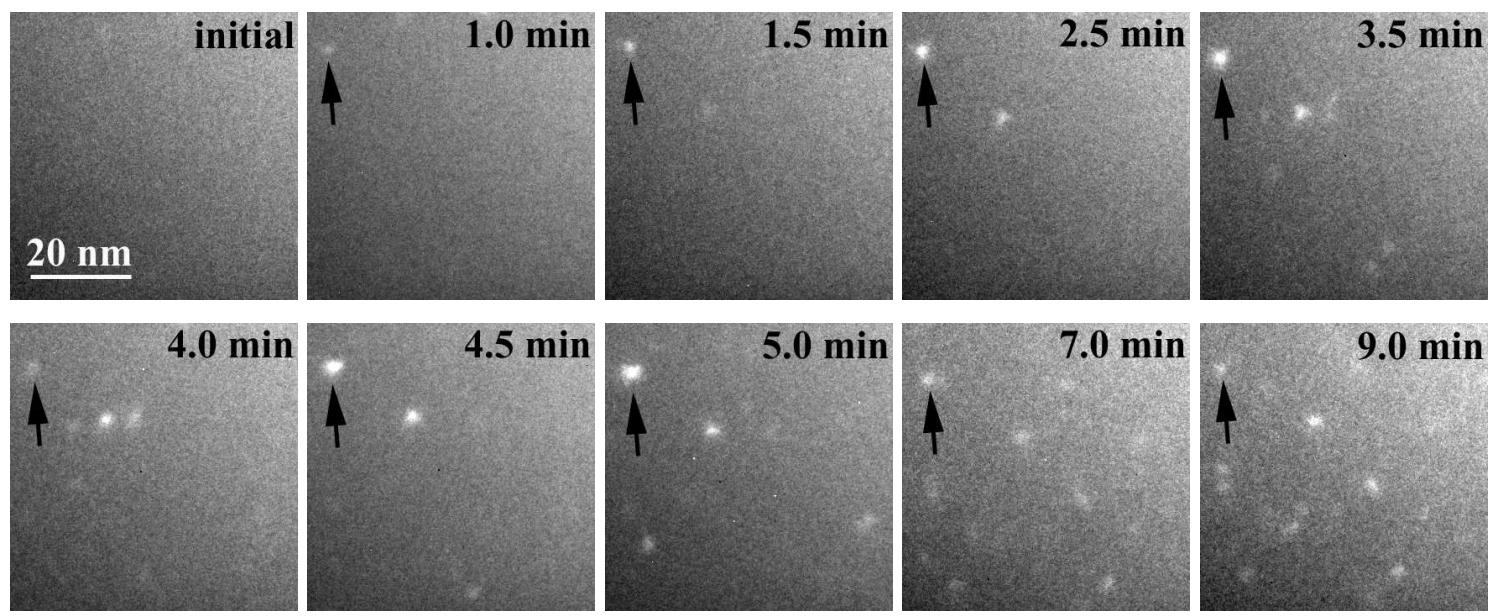

Figure 2 In situ dark-field TEM image of the $3.4 \mathrm{Na}_{2} \mathrm{O}-3.3 \mathrm{~K}_{2} \mathrm{O}-13.3 \mathrm{Ga}_{2} \mathrm{O}_{3}-80.0 \mathrm{SiO}_{2}$ glass during electron irradiation. 